\title{
Nodule Regression in Adults With Nodular Gastritis
}

\author{
Ji Wan Kimª, Sun-Young Lee ${ }^{\mathrm{a}, \mathrm{c}}$, Jeong Hwan Kimª, In-Kyung Sung ${ }^{\mathrm{a}}$, Hyung Seok Park ${ }^{\mathrm{a}}$, \\ Chan-Sup Shima ${ }^{a}$, Hye Seung Han ${ }^{\text {b }}$
}

\begin{abstract}
Background: Nodular gastritis (NG) is associated with the presence of Helicobacter pylori infection, but there are controversies on nodule regression in adults. The aim of this study was to analyze the factors that are related to the nodule regression in adults diagnosed as NG.
\end{abstract}

Methods: Adult population who were diagnosed as NG with $H$. pylori infection during esophagogastroduodenoscopy (EGD) at our center were included. Changes in the size and location of the nodules, status of $H$. pylori infection, upper gastrointestinal (UGI) symptom, EGD and pathology findings were analyzed between the initial and follow-up tests.

Results: Of the 117 NG patients, $66.7 \%(12 / 18)$ of the eradicated NG patients showed nodule regression after $H$. pylori eradication, whereas $9.9 \%(9 / 99)$ of the non-eradicated NG patients showed spontaneous nodule regression without $H$. pylori eradication $(\mathrm{P}<0.001)$. Nodule regression was more frequent in NG patients with antral nodule location $(\mathrm{P}=0.010)$, small-sized nodules $(\mathrm{P}=0.029)$, H. pylori eradication $(\mathrm{P}<0.001)$, UGI symptom $(\mathrm{P}=0.007)$, and a long-term follow-up period $(\mathrm{P}=0.030)$. On the logistic regression analysis, nodule regression was inversely correlated with the persistent $H$. pylori infection on the follow-up test (odds ratio (OR): $0.020,95 \%$ confidence interval $(\mathrm{CI}): 0.003-0.137, \mathrm{P}<0.001)$ and short-term followup period $<30.5$ months (OR: $0.140,95 \%$ CI: $0.028-0.700, \mathrm{P}=$ 0.017).

Conclusions: In adults with NG, H. pylori eradication is the most significant factor associated with nodule regression. Long-term follow-up period is also correlated with nodule regression, but is less significant than $H$. pylori eradication. Our findings suggest that $H$. pylori eradication should be considered to promote nodule regression

Manuscript accepted for publication November 06, 2015

aDepartment of Internal Medicine, Konkuk University School of Medicine, Seoul, Korea

bDepartment of Pathology, Konkuk University School of Medicine, Seoul, Korea

${ }^{\mathrm{c} C o r r e s p o n d i n g ~ A u t h o r: ~ S u n-Y o u n g ~ L e e, ~ D e p a r t m e n t ~ o f ~ I n t e r n a l ~ M e d i c i n e, ~}$ Konkuk University School of Medicine, 120-1 Neungdong-ro, Gwangjin-gu, Seoul 143-729, Korea. Email: sunyoung@kuh.ac.kr

doi: http://dx.doi.org/10.14740/gr692w in NG patients with $H$. pylori infection.

Keywords: Helicobacter pylori; Eradication; Gastritis; Nodule; Regression

\section{Introduction}

Nodular gastritis (NG) is characterized by an unusual gooseflesh appearance on esophagogastroduodenoscopy (EGD). There is accumulating evidence that Helicobacter pylori infection plays a role in the pathogenesis of NG [1-3]. Most cases are diagnosed incidentally because they are asymptomatic [4], but some patients manifest atypical upper gastrointestinal (UGI) symptoms such as indigestion, nausea, abdominal bloating, epigastric pain or abdominal discomfort $[5,6]$. However, there is insufficient evidence to recommend a $H$. pylori testand-treat strategy as an initial management approach to Korean adults presenting as functional dyspepsia [6]. The prevalence of NG decreases with aging, and is higher in women than in men $[2,4,7]$. We found that the prevalence of NG in Korean adults was $52(2.0 \%)$ of 2,059 asymptomatic Korean adults [4].

There are two endoscopic patterns of nodules in NG, and the clinicopathological findings do not appear to differ significantly between the two patterns [7]. A granular-type consists of small nodules with a size $<5 \mathrm{~mm}$ exhibiting regular protrusion, while a nodular-type consists of larger nodules $(\geq 5$ $\mathrm{mm}$ ) exhibiting large uniform protrusions. With regard to the location of the nodules, NG can involve both the antrum and body, with a predominantly nodular-type pattern on the antrum $[1,3]$. In addition, the nodules on the antrum change from the diffuse-type NG to the nondiffuse-type NG with the progress of gastric atrophy [8].

The prognosis differs in adults with NG; some might progress to a diffuse-type gastric cancer and a few may progress to a lymphofollicular malignancy $[9,10]$. Several studies have found reductions in symptoms and in the risk of peptic ulcers and possibly gastric cancer following $H$. pylori eradication [5, $11,12]$. Nevertheless, the characteristics of NG patients who show nodule regression are still uncertain. The aim of the present study was to identify the factors related to the improvement of NG in adults with $H$. pylori infection. 


\section{Patients and Methods}

\section{Study population}

This cross-sectional study included Korean adults who were diagnosed as NG with $H$. pylori infection at our center between August 2005 and April 2013. NG patients were included when EGD was followed up at our center after the initial diagnosis. NG patients were excluded when the age was below 20 years old, had a history gastric surgery, or had other significant disease at the time of diagnosis. Furthermore, those without preserved gastric biopsied specimens were also excluded from the study.

All of the included NG patients gave their written informed consent before EGD and answered to the questionnaires on their recent symptoms and medical history. This study was approved by the Institutional Review Board (IRB) of Konkuk University School of Medicine which confirmed that the study was in accordance with the ethical guidelines of the Helsinki Declaration. After the IRB approval at our center (KUH 1010574), it was registered as ClinicalTrials.gov ID: KCT0001148 (https://cris.nih.go.kr).

\section{Endoscopic examination}

EGD was performed with either EG-2990i endoscope (Pentax, Tokyo, Japan) or GIF-H260 endoscope (Olympus, Tokyo, Japan) at our center. Endoscopic findings of the background mucosa was classified as normal finding, chronic superficial gastritis, hemorrhagic gastritis, erosive gastritis, verrucous gastritis, chronic atrophic gastritis, metaplastic gastrtiis, and hypertrophic gastririts. Other notable findings such as nodular changes or hemorrhagic spots were recorded additionally.

\section{Diagnosis for NG}

NG was defined as diffuse chicken-skin-like mucosal changes or unusual goose-flesh apprearance on the endoscopic finding. When the nodules exhibit regular protrusion with a size $<5$ $\mathrm{mm}, \mathrm{NG}$ was subclassified as a small granular-type. When the nodules exhibit larger size with uniform protrusion, it was subclassified as a large nodular-type.

\section{Histopathology}

Gastric biopsied specimens were fixed in formalin. They were stained with hematoxylin and eosin (H\&E) and with modified Giemsa staining. Positive Giemsa staining was regarded as H. pylori infection. Based on the updated Sydney system, the grades were scored as either none, mild, moderate, or marked for chronic changes including atrophy and intestinal metaplasia $(0=$ none, $1=$ mild, $2=$ moderate, and $3=$ marked infiltration). In addition, the grades were scored for acute changes including activity (the intensity of acute polymorphonuclear cell infiltrates) and inflammation (the intensity of chronic mononuclear cell infiltrates).

\section{Diganosis and treatment for $H$. pylori infection}

The presence of $H$. pylori infection was determined when more than one of the invasive tests showed positive finding. For the invasive tests, histology and Giemsa stain were performed during EGD with or without the rapid urease test (CLO test; Roswell, USA manufactured by Korea Green Cross Medical Science, Eumseong, Korea).

NG patients were sent to the Digestive Disease Center if the patient agreed on further management. For the treatment, $H$. pylori eradication was performed using a triple therapy with a proton pump inhibitor (PPI, standard dose twice), amoxicillin (1 g twice) and clarithromycin (500 mg twice) daily for 1 week according to the guideline [13]. If the initial eradication has failed, a quadrauple therapy was performed with a PPI (standard dose twice), bismuth (120 mg four times), tetracycline (500 mg four times), and metronidazole (500 mg thrice) daily for 1 week.

\section{Follow-up tests}

After the initial diagnosis, NG patients underwent EGD and $H$. pylori test at our center as the initial tests. Changes in the size and location of the nodules, status of $H$. pylori infection, UGI symptom, EGD and pathology findings were compared with the previous findings by one gastroenterologist (Dr. J. W. Kim) to exclude any interobserver variability. Improvement of $\mathrm{NG}$ was defined as nodule regression on EGD findings.

\section{Statistical analysis}

Continuous variables were analyzed using the Student's $t$-test and were expressed as the mean \pm standard deviation (SD) values. Variables exhibiting skewed distributions were expressed as median values with ranges (minimum and maximum values), and differences were tested using the Kruskal-Wallis test. Categorical variables were analyzed using the Chi-square test and were presented as percentage. For the cut-off value of follow-up periods, a receiver operating characteristic (ROC) curve was constructed by plotting sensitivity (true-positive rate) against 1-specificity (false-positive rate) over all possible threshold levels of follow-up periods that were found to be related to the nodule regression. Based on the area under the ROC curve (AUC) analysis with $95 \%$ confidence interval (CI) and standard error (SE) values, the accuracy of the follow-up period for detecting nodule regression was caluculated. With the cut-off value provided by the ROC curve analysis, logistic regression analysis was performed to identify variables that were associated with nodule regression. Odds ratio (OR) and 95\% CI were analyzed for each significant variable. All analyses were performed using SPSS software, Windows version 19.0. A P-value of less than 0.05 was considered to be statisti- 
Table 1. Baseline Characteristics of the Included Subjects

\begin{tabular}{|c|c|}
\hline Characteristics & NG patients $(n=117)$ \\
\hline Age (years, mean $\pm \mathrm{SD}$ ) & $38.3 \pm 8.0$ \\
\hline Gender (female, \%) & $86(73.5 \%)$ \\
\hline Follow-up period (months, mean $\pm \mathrm{SD}$ ) & $32.0 \pm 14.7$ \\
\hline \multicolumn{2}{|l|}{ Presence of UGI symptom (\%) } \\
\hline Epigastric pain & $8(6.8 \%)$ \\
\hline Indigestion & $5(4.3 \%)$ \\
\hline Abdominal distention & $2(1.7 \%)$ \\
\hline Nausea & $2(1.7 \%)$ \\
\hline \multicolumn{2}{|l|}{ Size of the nodules $(\%)$} \\
\hline Small granular-type & $64(54.7 \%)$ \\
\hline Large nodular-type & $53(45.3 \%)$ \\
\hline \multicolumn{2}{|l|}{ Location of the nodules (\%) } \\
\hline Antrum only & $73(62.4 \%)$ \\
\hline Body involved & $44(37.6 \%)$ \\
\hline Presence of hemorrhagic spots (\%) & $21(17.9 \%)$ \\
\hline H. pylori eradication $(\%)$ & $18(15.4 \%)$ \\
\hline \multicolumn{2}{|l|}{ Pathology based on the updated Sydney system } \\
\hline Inflammation (none/mild/moderate/marked) & $0: 5: 91: 21$ \\
\hline Activity (none/mild/moderate/marked) & $5: 42: 61: 9$ \\
\hline Atrophy (none/mild/moderate/marked) & $91: 22: 4: 0$ \\
\hline Intestinal metaplasia (none/mild/moderate/marked) & 106:10:0:1 \\
\hline H. pylori (none/mild/moderate/marked) & $6: 10: 48: 53$ \\
\hline
\end{tabular}

SD: standard deviation; UGI: upper gastrointestinal.

cally significant.

\section{Results}

\section{Baseline characteristics of the NG patients}

A total of $117 \mathrm{NG}$ patients fulfilled the study criteria and underwent follow-up EGD at our center after the initial diagnosis of NG. Most of the NG patients $(85.5 \%)$ did not have UGI symptom at the time of endoscopic examination (Table 1).

\section{Follow-up tests with or without $H$. pylori eradication}

Of the included NG patients, 18 (15.4\%) underwent $H$. pylori eradication after the diagnosis. At the follow-up tests, none of the eradicated patients showed $H$. pylori infection, whereas all of the non-eradicated patients showed persistent H. pylori infection. Nodule regression was noticed at the follow-up tests in $66.7 \%$ of the 18 eradicated NG patients after $H$. pylori eradication, whereas it was noticed in $9.9 \%$ of the 99 non-eradicated NG patients $(\mathrm{P}<0.001)$. None of these patients progressed to gastric cancer during the follow-up period.

\section{Variables correlated with nodule regression}

Using the correlation analysis, follow-up period $(\mathrm{r}=-0.200, \mathrm{P}$ $=0.030)$, UGI symptom $(\mathrm{r}=-0.250, \mathrm{P}=0.007)$, nodule size $(\mathrm{r}$ $=0.202, \mathrm{P}=0.029)$ and $H$. pylori eradication $(\mathrm{r}=-0.541, \mathrm{P}<$ 0.001 ) were related to nodule regression (Table 2). When the patients were subclassified into two groups according to the presence of nodules at the follow-up tests, nodule regression was related to the presence of UGI symptom $(\mathrm{P}=0.007), H$. pylori eradication $(\mathrm{P}<0.001)$, and small size $(\mathrm{P}=0.029)$ of the nodules (Table 3 ).

Furthermore, NG patients with nodule regression showed a longer follow-up period $(38.2 \pm 12.1$ months $)$ than those without nodule regression $(30.6 \pm 14.9$ months, $\mathrm{P}=0.030)$. Using the ROC analysis, the cut-off value of follow-up period on nodule regression was 30.5 months (AUC: $0.651,95 \% \mathrm{CI}$ : $0.540-0.763$, SE: $0.057, \mathrm{P}=0.030)$ with a sensitivity of $71.4 \%$ and specificity of $53.1 \%$.

\section{Importance of $\boldsymbol{H}$. pylori eradication and follow-up period on nodule regression}

Of all significant variables, only $H$. pylori eradication and 
Table 2. Correlation Analysis of the Variables on Nodule Regression

\begin{tabular}{lll}
\hline Variables & Correlation coefficient & P-value \\
\hline Age & 0.044 & 0.637 \\
Gender & 0.072 & 0.437 \\
Follow-up period & -0.200 & 0.030 \\
Presence of upper gastrointestinal symptom & -0.250 & 0.007 \\
Size of the nodules & 0.202 & 0.029 \\
Location of the nodules & 0.133 & 0.152 \\
Presence of hemorrhagic spots & 0.103 & 0.271 \\
H. pylori eradication & -0.541 & $<0.001$ \\
Pathology findings based on the updated Sydney system & & 0.127 \\
$\quad$ Inflammation & 0.142 & 0.345 \\
$\quad$ Activity & -0.088 & 0.773 \\
$\quad$ Atrophy & -0.027 & 0.099 \\
$\quad$ Intestinal metaplasia & -0.153 & 0.899 \\
\hline H. pylori infiltration & -0.012 & \\
\hline
\end{tabular}

follow-up period were significant on the logistic regression analysis (Table 4). Nodule regression was inversely correlated with the persistent $H$. pylori infection on the follow-up test (OR: $0.020,95 \%$ CI: $0.003-0.137, \mathrm{P}<0.001$ ) and short-term follow-up period $<30.5$ months (OR: $0.140,95 \% \mathrm{CI}$ : 0.028 - 0.700, $\mathrm{P}=0.017$ ). Conversely, persistent nodules in NG patients were significantly higher in the non-eradicated group (OR: 49.948, 95\% CI: 7.274 - 342.957, $\mathrm{P}<0.001$ ) and short- term follow-up period $<30.5$ months (OR: 7.132, 95\% CI: 1.430 - 35.581, $\mathrm{P}=0.017$ ).

\section{Subgroup analysis according to $H$. pylori eradication}

Of $99 \mathrm{NG}$ patients without eradication, nine (9.1\%) showed spontaneous nodule regression. Between these nine NG pa-

Table 3. Comparison Between the Patients With Nodule Regression and Those With Persistent Nodules at the Follow-Up Tests

\begin{tabular}{|c|c|c|c|}
\hline Variables & $\begin{array}{l}\text { Patients with nodule } \\
\text { regression }(n=21)\end{array}$ & $\begin{array}{l}\text { Patients with persistent } \\
\text { nodules }(\mathrm{n}=96)\end{array}$ & P-value \\
\hline Age (years, mean $\pm \mathrm{SD}$ ) & $37.6 \pm 8.5$ & $38.5 \pm 7.9$ & 0.637 \\
\hline Gender (female, \%) & $14(66.7 \%)$ & $72(75.0 \%)$ & 0.433 \\
\hline Presence of UGI symptom (\%) & $7(33.3 \%)$ & $10(10.4 \%)$ & 0.007 \\
\hline Size of the nodules $(\%)$ & & & 0.029 \\
\hline Small granular-type & $16(76.2 \%)$ & $48(50.0 \%)$ & \\
\hline Large nodular-type & $5(23.8 \%)$ & $48(50.0 \%)$ & \\
\hline Location of the nodules (\%) & & & 0.214 \\
\hline Antrum only & $16(76.2 \%)$ & $57(59.4 \%)$ & \\
\hline Body involved & $5(23.8 \%)$ & $39(40.6 \%)$ & \\
\hline Presence of hemorrhagic spots (\%) & $2(9.5 \%)$ & $19(19.8 \%)$ & 0.267 \\
\hline \multicolumn{4}{|l|}{ Pathology scores $(0: 1: 2: 3)$} \\
\hline Inflammation & $0: 2: 17: 2$ & $0: 3: 74: 19$ & 0.260 \\
\hline Activity & $0: 7: 12: 2$ & $5: 35: 49: 7$ & 0.711 \\
\hline Atrophy & $17: 2: 2: 0$ & $74: 20: 2: 0$ & 0.136 \\
\hline Intestinal metaplasia & 18: $2: 0: 1$ & $88: 8: 0: 0$ & 0.097 \\
\hline H. pylori & 1:1:10:9 & $5: 9: 38: 44$ & 0.864 \\
\hline H. pylori eradication (\%) & $12(57.1 \%)$ & $6(6.2 \%)$ & $<0.001$ \\
\hline Follow-up period (months, mean $\pm \mathrm{SD}$ ) & $38.2 \pm 12.1$ & $30.6 \pm 14.9$ & 0.030 \\
\hline
\end{tabular}

SD: standard deviation; UGI: upper gastrointestinal. 
Table 4. Significant Variables Related to Nodule Regression

\begin{tabular}{|c|c|c|c|}
\hline Variables & Odds ratio & $95 \%$ confidence interval & P-value \\
\hline \multicolumn{4}{|l|}{ H. pylori eradication } \\
\hline No (persistent $H$. pylori infection) & 0.020 & $0.003-0.137$ & $<0.001$ \\
\hline Yes & 1 (reference) & & \\
\hline \multicolumn{4}{|l|}{ Follow-up period* } \\
\hline$<30.5$ months & 0.140 & $0.028-0.700$ & 0.017 \\
\hline$\geq 30.5$ months & 1 (reference) & & \\
\hline \multicolumn{4}{|l|}{ Upper gastrointestinal symptom } \\
\hline Present & 1.483 & $0.246-8.936$ & 0.667 \\
\hline Absent & 1 (reference) & & \\
\hline \multicolumn{4}{|l|}{ Size of the nodules } \\
\hline Small & 2.670 & $0.738-9.658$ & 0.134 \\
\hline Large & 1 (reference) & & \\
\hline
\end{tabular}

${ }^{*}$ Cut-off value was determined by the ROC curve analysis.

tients with nodule regression and other $90 \mathrm{NG}$ patients with persistent nodules, there were no significant differences on age $(P=0.605)$, gender $(P=0.224)$, nodule size $(P=0.165)$, location $(\mathrm{P}=0.279)$, UGI symptom $(\mathrm{P}=0.502)$, hemorrhagic spots $(\mathrm{P}=0.351)$, and follow-up period $(\mathrm{P}=0.131)$. With regard to the scores based on the updated Sydney system, there were no significant differences on inflammation $(\mathrm{P}=0.351)$, activity $(\mathrm{P}$ $=0.824)$, atrophy $(\mathrm{P}=0.094)$, and $H$. pylori infiltration $(\mathrm{P}=$ 0.880 ) scores, but median intestinal metaplasia score was significantly higher in those with spontaneous nodule regression (0.25 ranging from 0 to 3 ) than those without regression $(0.08$ ranging from 0 to $1, \mathrm{P}=0.008$ ).

All the eradicated patients exhibited no $H$. pylori at the follow-up tests, and 12 (66.7\%) showed nodule regression. Between these $12 \mathrm{NG}$ patients with nodule regression and other six NG patients with persistent nodules, there were no significant differences on age $(\mathrm{P}=0.944)$, gender $(\mathrm{P}=0.344)$, nodule size $(\mathrm{P}=0.294)$, location $(\mathrm{P}=0.073)$, $\mathrm{UGI}$ symptom $(\mathrm{P}=0.563)$ and hemorrhagic spots $(\mathrm{P}=0.569)$. Mean follow-up period of the 12 improved NG patients (37.6 \pm 12.6 months) was significantly longer than that of the six persistent NG patients $(21.8 \pm$ 6.5 months, $\mathrm{P}=0.012)$. On the pathology findings, there were no significant differences on activity $(\mathrm{P}=0.258)$, atrophy $(\mathrm{P}=$ $0.201)$, intestinal metaplasia $(\mathrm{P}=0.621)$, and $H$. pylori infiltration $(\mathrm{P}=0.285)$, but mean inflammation score was significantly higher in $\mathrm{NG}$ patients with persistent nodules $(2.3 \pm 0.5)$ than in those with nodule regression $(2.0 \pm 0.0, \mathrm{P}=0.035)$.

\section{Discussion}

This study found that H. pylori eradication is the most important factor related to nodule regression in NG. In addition, longer follow-up period was a significant independent factor for nodule regression in NG patients with $H$. pylori eradication. Neither the gender nor the age of the patient was related to the prognosis of $\mathrm{NG}$, despite $\mathrm{NG}$ being more common in young women.

In this study, two-thirds of the eradicated NG patients exhibited complete improvement of $\mathrm{NG}$ at the follow-up tests. Similarly, endoscopic evidence of $\mathrm{NG}$ disappeared in $63.2 \%$ of the NG patients at 2 months after $H$. pylori eradication in a Chinese study [11]. The observation of nodule regression after eradication in the present study supports the recommendation that $H$. pylori eradication should be performed to the adults with $\mathrm{NG}$ for nodule regression $[5,11,12]$. Notably, such improvements do not occur immediately, and regression requires a substantial amount of time [8]. This study exhibited that longer follow-up period is also correlated with the improvement of $\mathrm{NG}$, but follow-up period was less significant than eradication. Our findings suggest that longer follow-up period is a significant independent factor for nodule regression in $\mathrm{NG}$ patients, but it is less accurate when used alone for predicting nodule regression without eradication.

Interestingly, $9.1 \%$ of the NG patients without $H$. pylori eradication in the present study exhibited spontaneous regression of the nodules, even with persistent $H$. pylori infection. Few studies have found a tendency for antral nodules to regress more frequently than those on the body $[1,3]$. This finding may be attributable to differences in the severity and depth of $H$. pylori-induced inflammation on the antrum and body, and because antral nodules tend to regress in association with atrophic changes $[8,14]$. Although the correlation between $\mathrm{NG}$ and UGI symptom is still uncertain [6], nodule regression was more frequent in NG patients with UGI syptoms in our study, because those with symptoms tend to undergo $H$. pylori eradication. The presence of UGI symptom was not an idependent signficant factor for nodule regression in this study.

With regard to nodule size, small ones (granular-type $\mathrm{NG}$ ) tended to regress more often than the large ones (nodular-type NG) in our NG patients. This can be explained by the histological findings, whereby smaller lymphoid follicles resolve more easily than large lymphoid follicles with other inflammatory cells. Although we were unable to determine why the smaller 
antral nodules regressed more readily than the large body nodules, we suggest that this is due to the different proportions of histopathological features, including the presence of gastric atrophy, intestinal metaplasia, lymphoid aggregates, or eosinophil infiltrates in the mucosal layer, as shown previously [15]. The histopathological features of NG differ according to the size and location of the nodules, and thus improvement after eradication will differ based on these factors.

This study found that pathology findings could be related to nodule regression. In case of eradicated NG patients, those with persistent nodules even after the eradication exhibited higher inflammation score. Our findings suggest that higher intensity of chronic mononuclear cell infiltrates in these $\mathrm{NG}$ patients might interfere nodule regression by continous aggregation of the lymphoid follicles. On the other hand, in case of non-eradicated NG patients, those with spontaneous nodule regression exhibited higher intestinal metaplasia score. This is consistent with previous studies that NG improves upon senile changes of the gastric mucosa such as atrophic gastriits and intestinal metaplasia [7-9, 14].

There are several limitations in this study. Firstly, H. $p y$ lori eradication was not performed in all of the NG patients, because it is not covered by insurance in Korea. It was performed only in NG patients who agreed on additional payment for eradication. Secondly, there was no malignant change in all NG patients at the follow-up tests. Therefore, we could not find a correlation between NG and gastric malignancy in this study. Despite all these limitations, there was a significant difference on nodule regression between the NG patients with $H$. pylori eradication and those without eradication.

\section{Conclusions}

H. pylori eradication is the most significant factor associated with the prognosis of NG in adults. Although there is a tendency for NG patients with longer follow-up to regress nodules, the significance as a prognostic factor for nodule regression is low when compared to that of $H$. pylori eradication. Together, the findings of this study suggest that adults with NG should undergo $H$. pylori eradication to promote nodule regression regardless of the size and location of the nodules.

\section{Acknowledgement}

This work was supported by Konkuk University.

\section{Conflict of Interest}

This study was supported by Konkuk University in 2015 (KU2015-A019-0270).

\section{References}

1. Chen MJ, Wang TE, Chang WH, Liao TC, Lin CC, Shih
SC. Nodular gastritis: an endoscopic indicator of Helicobacter Pylori infection. Dig Dis Sci. 2007;52(10):26622666.

2. Choi HJ, Lee SY, Lee JH, Seol DC, Kim SY, Choi HJ, Park HS, et al. Two atypical cases of nodular gastritis: a poorly differentiated gastric adenocarcinoma and a pseudo-low grade gastric MALT lymphoma. Gastroenterol Res. 2010;3(1):41-45.

3. Al-Enezi SA, Alsurayei SA, Aly NY, Ismail AE, Ismail WA, Al-Brahim N, El-Dousari A. Endoscopic nodular gastritis in dyspeptic adults: prevalence and association with Helicobacter pylori infection. Med Princ Pract. 2010;19(1):40-45.

4. Hong SN, Jo S, Jang JH, Choi J, Kim S, Ahn SY, Kim JH, et al. Clinical characteristics and the expression profiles of inflammatory cytokines/cytokine regulatory factors in asymptomatic patients with nodular gastritis. Dig Dis Sci. 2012;57(6):1486-1495.

5. Dwivedi M, Misra SP, Misra V. Nodular gastritis in adults: clinical features, endoscopic appearance, histopathological features, and response to therapy. J Gastroenterol Hepatol. 2008;23(6):943-947.

6. Lee H, Jung HK, Huh KC. Current status of functional dyspepsia in Korea. Korean J Intern Med. 2014;29(2):156165.

7. Nakashima R, Nagata N, Watanabe K, Kobayakawa M, Sakurai T, Akiyama J, Hoshimoto K, et al. Histological features of nodular gastritis and its endoscopic classification. J Dig Dis. 2011;12(6):436-442.

8. Shiotani A, Kamada T, Kumamoto M, Nakae Y, Nakamura Y, Kakudo K, Haruma K. Nodular gastritis in Japanese young adults: endoscopic and histological observations. J Gastroenterol. 2007;42(8):610-615.

9. Kitamura S, Yasuda M, Muguruma N, Okamoto K, Takeuchi H, Bando Y, Miyamoto H, et al. Prevalence and characteristics of nodular gastritis in Japanese elderly. J Gastroenterol Hepatol. 2013;28(7):1154-1160.

10. Kamada T, Tanaka A, Yamanaka Y Manabe N, Kusunoki H, Miyamoto M, Tanaka S, et al. Nodular gastritis with Helicobacter pylori infection is strongly associated with diffuse-type gastric cancer in young patients. Dig Endosc. 2007;19(1):180-184.

11. Chen MJ, Shih SC, Wang TE, Chan YJ, Chen CJ, Chang WH. Endoscopic patterns and histopathological features after eradication therapy in Helicobacter pylori-associated nodular gastritis. Dig Dis Sci. 2008;53(7):18931897.

12. Shimatani T, Inoue M, Iwamoto K, Hyogo H, Yokozaki M, Saeki T, Tazuma S, et al. Gastric acidity in patients with follicular gastritis is significantly reduced, but can be normalized after eradication for Helicobacter pylori. Helicobacter. 2005;10(3):256-265.

13. Kim SG, Jung HK, Lee HL, Jang JY, Lee H, Kim CG, Shin $\mathrm{WG}$, et al. Guidelines for the diagnosis and treatment of Helicobacter pylori infection in Korea, 2013 revised edition. J Gastroenterol Hepatol. 2014;29(7):13711386.

14. Ricuarte O, Gutierrez O, Cardona H, Kim JG, Graham DY, El-Zimaity HM. Atrophic gastritis in young children 
and adolescents. J Clin Pathol. 2005;58(11):1189-1193.

15. Sakaguchi M, Amemoto K, Honda M, Hashimoto T, Asakuma Y, Shimada M, Yamamoto N, et al. Clinical evalu- ation of nodular gastritis: follow-up by endoscopy and histopathology. Gastroenterol Endosc. 2006;48(10):24772485. 